\title{
Carbon/proton therapy: A novel gantry design
}

\author{
D. Trbojevic* and B. Parker \\ Brookhaven National Laboratory, Upton, New York 11973, USA \\ E. Keil \\ CERN, Geneva, Switzerland
}

A. M. Sessler

Lawrence Berkeley National Laboratory, Berkeley, California 94720, USA

(Received 7 September 2006; published 29 May 2007)

\begin{abstract}
A major expense and design challenge in carbon/proton cancer therapy machines are the isocentric gantries. The transport elements of the carbon/proton gantry are presently made of standard conducting dipoles. Because of their large weight, of the order of $\sim 100$ tons, the total weight of the gantry with support structure is $\sim 600$ tons. The novel gantry design that is described here is made of fixed field superconducting magnets, thus dramatically reducing magnet size and weight compared to conventional magnets. In addition, the magnetic field is constant throughout the whole energy region required for tumor treatment. Particles make very small orbit offsets, passing through the beam line. The beam line is built of combined-function dipoles such as a nonscaling fixed field alternating gradient (NS-FFAG) structure. The very large momentum acceptance NS-FFAG comes from very strong focusing and very small dispersion. The NS-FFAG small magnets almost completely filled the beam line. They first make a quarter (or close to a quarter) of an arc bending upward and an additional half of a circle beam line finishing so that the beam is pointed towards the patient. At the end of the gantry, additional magnets with a fast response are required to allow radial scanning and to provide the required position and spot size. The fixed field combined-function magnets for the carbon gantry could be made of superconducting magnets by using low temperature superconducting cable or by using high temperature superconductors.
\end{abstract}

DOI: 10.1103/PhysRevSTAB.10.053503

PACS numbers: 29.27.Eg, 41.85.Ja, 41.75.Ak

\section{INTRODUCTION}

The number of hadron cancer therapy facilities is continuously growing all around the world due to many advantages with respect to the already existing $\mathrm{x}$-ray treatments (using electron accelerators). New facilities are being commissioned or are in the process of being built $[1,2]$. The advantages are in much more localized effects with much smaller influence on surrounding healthy cells. Carbon therapy has advantages in specific cancer tumors with respect to the proton therapy. The new facilities, as the recent one in Heidelberg [3], combine the treatment with different ions: ranging from protons to oxygen. A major challenge in present and future hadron therapy facilities is the beam delivery system of the isocentric gantry. The ion dose has to be delivered to the patients from various positions, and with very good reliability and stability. Large cancerous tumors have to be hit both transversely and longitudinally. The ion positions are transversely varied by the scanning magnets, while the different beam energies define longitudinal positions of the Bragg peak. In addition, an angle variation around the patient is provided by the gantry rotation.

\footnotetext{
*Electronic address: dejan@bnl.gov
}

We describe the use of the principle of nonscaling fixed field alternating gradient (NS-FFAG) (previously reported [4]), with very strong focusing and small dispersion, to obtain a new way of precisely transporting ions to the patients. The relatively large number of small combinedfunction magnets with fixed magnetic field and linear gradients tightly control the ion transport. The required momentum range for the gantry depends on the position and size of the tumor; $\sim \pm 30 \%$ is reasonable. More details about the NS-FFAG concept and solutions for hadron acceleration for medical facilities are described elsewhere [5]. The weight of the gantry and support structure in the latest design of the Heidelberg carbon/proton facility is of the order of 630 tons. The transport components make 135 tons [3] while the rest is support and counterweight. The NS-FFAG concept provides reduction of the transport elements' weight by 2 orders of magnitude to about 1.5 tons. This is accomplished due to very small orbit offsets within the magnets, about $\pm 13.6 \mathrm{~mm}$. The presented gantry operates at fixed magnetic field for fully stripped carbon ions in the kinetic energy range of $150-400 \mathrm{MeV} / u$ with magnets set for the momentum rigidity of $B \rho=4.882 \mathrm{Tm}$. The same gantry could be used for the proton therapy with kinetic energy range of 90-250 MeV by adjusting magnetic field values for the momentum rigidity of $B \rho=1.87087 \mathrm{~T} \mathrm{~m}$. 


\section{THE NONSCALING FFAG}

The known principle of scaling FFAG accelerators [68] has recently been revived in Japan [9]. The positive and negative radial bending of the fixed field nonlinear combined-function magnets provides stable radial and vertical betatron oscillations over a very large energy range. In a radial sector FFAG the magnetic field varies transversely as $B \sim\left(r / r_{o}\right)^{k}$ with $r$ as the radial offset and $r_{o}$ as the reference radius. The $k$ value could be very large $k \sim 1000$ and the negative bend is a third of the major bend. The orbits at different energies are parallel to each other with fixed tunes and chromaticities. The central orbit is presented in Fig. 2 in the referenced article [8].

The NS-FFAG follows essentially the important principles of the scaling design, mentioned above, with the exception that the stringent conditions of magnetic field dependence along the radial axis and placement of magnets are removed. The most important feature of the nonscaling FFAG gantry application is that the orbit offsets are 1 order of magnitude smaller than for the scaling one. Such a reduction of the magnet size would not be possible by using the principle of the scaling FFAG. This difference comes mostly from the triplet configuration: horizontally focusing then defocusing and again focusing instead of defocusing-focusing-defocusing as in the case of scaling design. There is similarity of the triplet NS-FFAG to minimum emittance lattices for synchrotron light sources where it is required to have the minima of the horizontal amplitude function and the dispersion at the center of the major bending elements. This provides a minimum for the average of dispersive function $\langle\mathcal{H}\rangle$ throughout the dipole:

$$
\langle\mathcal{H}\rangle=\frac{1}{L} \int_{0}^{L}\left(\gamma D^{2}+2 \alpha D D^{\prime}+\beta D^{\prime 2}\right) \mathrm{d} s .
$$

Here the standard orbit functions $\alpha, \beta, \gamma$, the dispersion $D$, and its derivative $D^{\prime}$ are all functions of $s$.

\section{A. The basic cell of NS-FFAG gantry}

Ion acceleration in the present carbon/proton therapy facilities is provided by either synchrotrons (Heidelberg, Loma Linda, etc.), cyclotrons (Massachusetts General Hospital, Accelerator Industrial Medical ApplicationAIMA, Nice, France), or scaling FFAGs (KEK). Energy variation is required for each treatment. The synchrotron provides energy variation by extracting at different field levels in the magnets. Presently, in any cancer therapy facility, the transport elements of the gantry have to have adjustable magnetic field for each energy. The elements of the gantry, presented in this report, for each kind of ion are always with fixed magnetic field in the whole required energy range. This feature should simplify operation. The ion orbits at different energies oscillate along the gantry with maximum offsets of the order of $18.9 \mathrm{~mm}$ (as shown in Fig. 1).

The principle of NS-FFAG was previously used to accelerate ions in the momentum range of the order of $\sim \pm 50 \%$. The acceleration requires relatively large drift space for $\mathrm{rf}$ cavity placement. The gantry is just a transfer line without any large drift requirements. Eliminating the drifts in the NS-FFAG further reduces the orbit offsets and makes it more compact. The central magnet is a horizon-

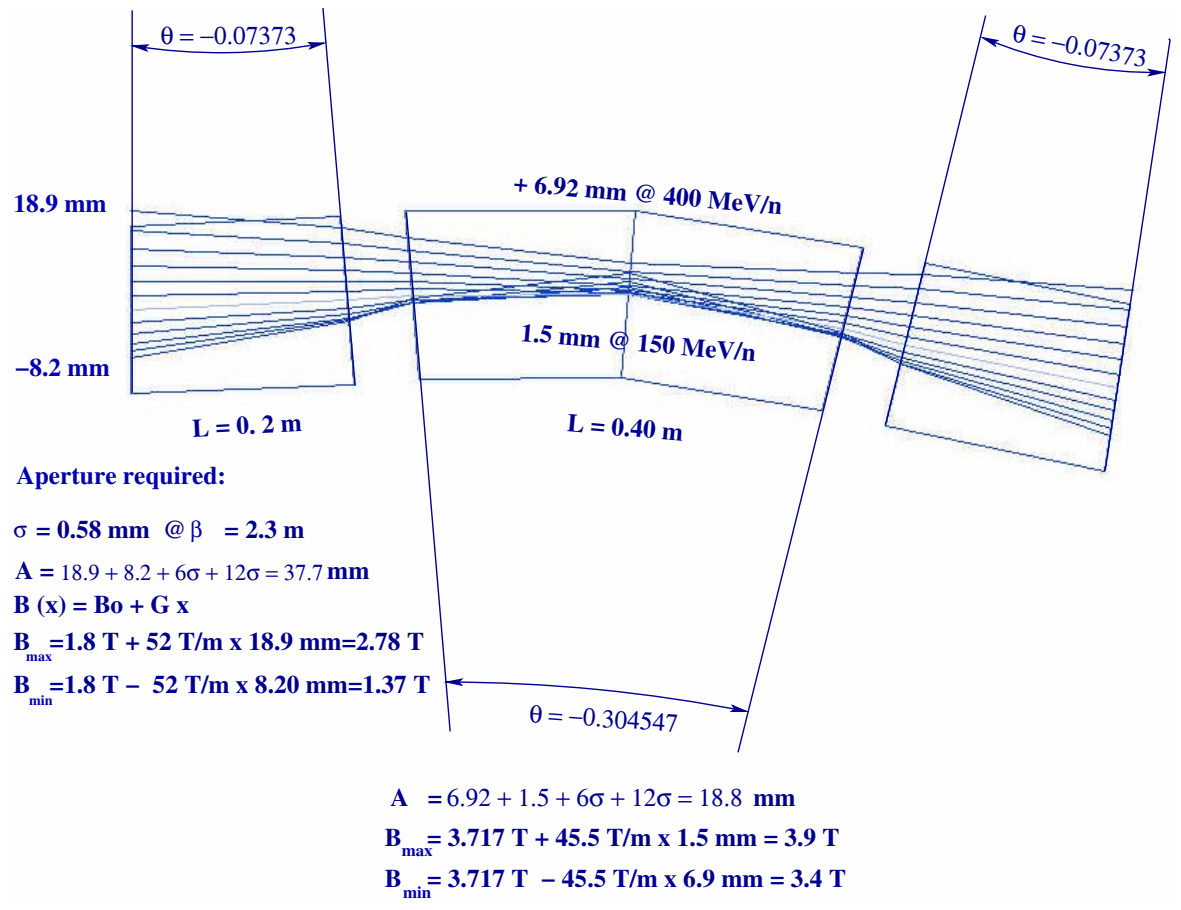

FIG. 1. (Color) Particle orbits in the basic gantry cell for a kinetic energy range of 149-400 MeV/u. 


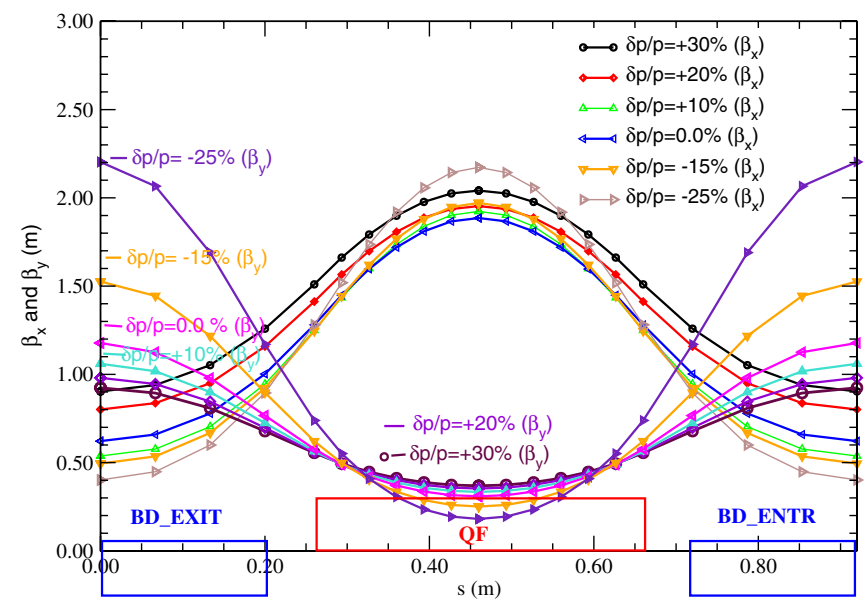

FIG. 2. (Color) Betatron functions and magnets in the basic cell.

tally defocusing combined-function magnet with minima of dispersion and horizontal $\beta_{x}$ function at the middle. The opposing bends on both sides are combined-function magnets with horizontally focusing linear gradient. The orbit offsets for the energy range of carbon ions of $149-400 \mathrm{MeV} / u$ are very small, about $\pm 5 \mathrm{~mm}$, in the middle of the major bend. The beginning and the end of the gantry, as well as the end of the first quarter of arc, are selected to be at the middle of the major bending element. The betatron functions of the basic cell are presented in Fig. 2. The dispersion throughout the NS-FFAG lattice retains very small values, contributing to the small momentum offsets. Since $\Delta x=D_{x} \delta p / p$, if the aperture is of the order of $\Delta x \approx 25 \mathrm{~mm}$ and dispersion is $D_{x} \approx 5 \mathrm{~cm}$, the momentum offset could be as much as $\delta p / p \approx 50 \%$. Additional focusing and scanning magnets correct small offsets at the end of the gantry. It should be noted that when the words horizontal and vertical are used it is assumed that the gantry is located horizontally. The meaning of these words is quite different as soon as the gantry is rotated out of the horizontal plane.

The gantry, made of the NS-FFAG cells, accepts and propagates different energy ions with very small variation of the orbit. The offsets at the end of the cell are obtained from the polymorphic tracking code [10] in a kinetic energy range $149.7-400 \mathrm{MeV} / u$ or in momentum $-25 \% \leq \delta p / p \leq 30 \%$. The dispersion and the slope are set to zero at the beginning of the gantry in the middle of the required momentum range. The largest orbit offsets are at the focusing quadrupoles, and have similar values at the lowest and highest momentum energy by optimization of the bending angles.

\section{B. Details of the gantry lattice design}

The NS-FFAG cell consists of two types of combinedfunction magnets: the major bend with horizontally defocusing gradient and the opposite bend with a focusing gradient. We use the middle of the major bend as the cell

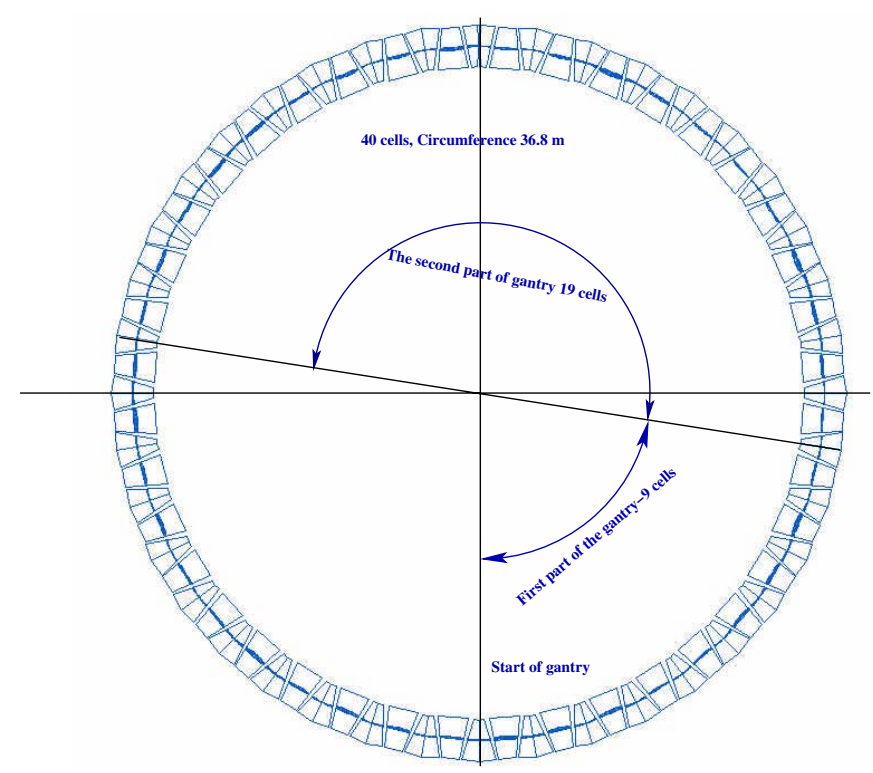

FIG. 3. (Color) The nonscaling FFAG, used for the gantry design. Orbit offsets are very small.

boundary. The half cell is then made of half of the major bend followed by a drift and half of the opposite bend with focusing gradient. The other half is its mirror image. This is now a standard FODO cell as the nonscaling FFAG accelerator becomes a transport beam line. The cell is designed by setting constraints at the middle of the momentum range: the minimum of the horizontal betatron function with slopes of dispersion and both betatron functions equal to zero at the middle of the major bend as presented in Fig. 3. The first step in gantry design is construction of a NS-FFAG ring without rf cavity drifts. Stable orbits for carbon ions are found within a momentum range of $-30 \% \leq \delta p / p \leq+30 \%$. The gantry NS-FFAG ring is presented in Fig. 3 .

Radial scanning and orbit correction is provided at the end of the gantry. The maximum height of the gantry (it is assumed now that the isocentric gantry is set within the plane normal to the patient plane) can be reduced if it is required by using a smaller number of cells in the first part.

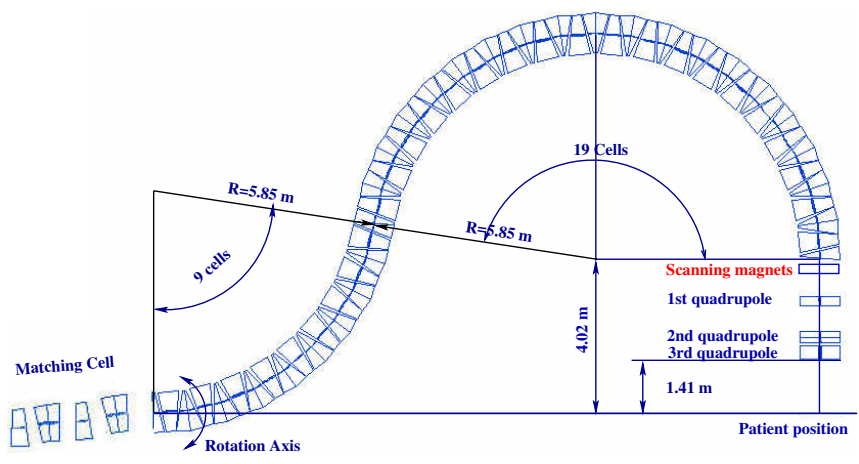

FIG. 4. (Color) Matching cell to the accelerator, the gantry, and the final focus with scanning magnets above the patient. 


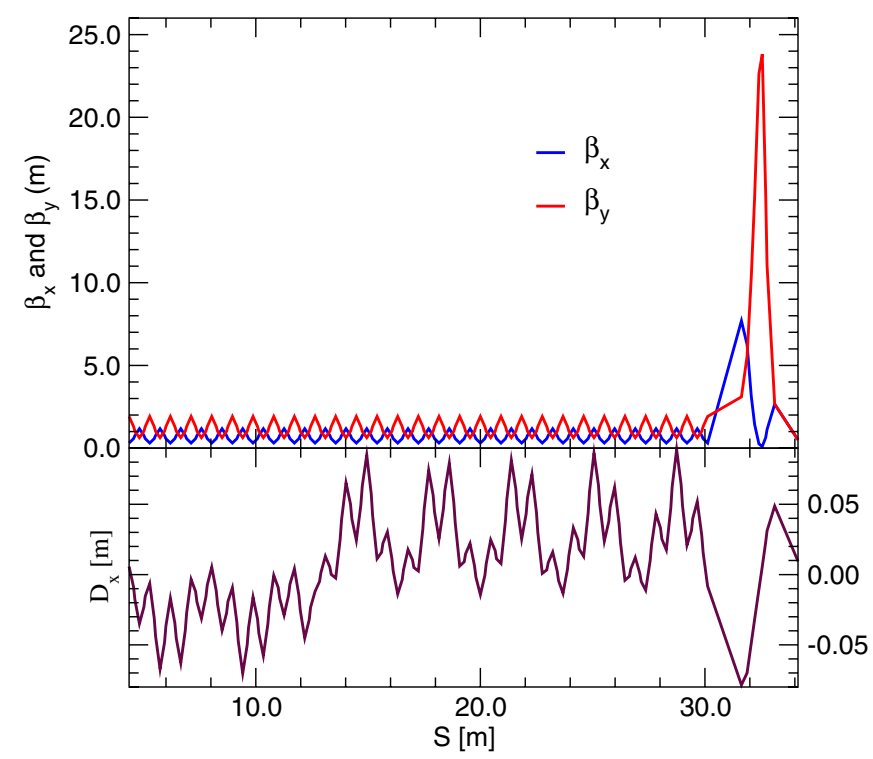

FIG. 5. (Color) Orbit functions in the gantry at the central momentum.

In the example presented in Fig. 4, the first section is made of 9 cells making a total bending angle of 81 degrees, followed by 19 cells (171-degree section) with opposite bending.

The orbit functions of the gantry together with the focusing triplet in front of the patient, at the central momentum, are shown in Fig. 5. The dispersion is not matched, because of the optimization described in Sec. II C.

\section{Optimization of the gantry parameters}

The goal of the gantry lattice optimization is to provide the smallest orbit offsets for all energies at the end of the gantry and to minimize the magnetic field value. Ray searches for both axes use input radii of $r_{x}, r_{y} \leq 0.5 \mathrm{~mm}$. The variables are: the relationship between the main and opposing bending fields, tunes per cell, magnet lengths, drift length, etc. The results of the bending field optimization are shown in Table I. The bending angles of $\theta_{Q F}=$ -0.0635 and $\theta_{B D}=0.284$, with the relationship between two angles as $\theta_{Q F} / \theta_{B D}=0.2235$, represent the optimum solution. This is set by the smallest values of maximum orbit offsets at the end of the gantry, namely: $x_{\min }=$ $-3.042 \mathrm{~mm}$ and $x_{\max }=4.227 \mathrm{~mm}$, making the smallest value of $\Delta x=7.269 \mathrm{~mm}$. The maximum orbit offset dependence on the relationship between the two bending angles $\theta_{Q F} / \theta_{B D}$ is shown in Fig. 6. Table I also shows that $D_{\min }$ vanishes at a slightly different ratio of $\theta_{Q F} / \theta_{B D}$.

\section{Matching of the gantry to the accelerator}

The horizontal betatron function and dispersion have very small values at the entrance to the gantry. In addition, the slopes of all orbit functions are equal to zero. To simplify the matching and operation, an additional $0.3 \mathrm{~m}$ long straight matching cell is added in front of the gantry entrance. The matching cell consists of two quadrupoles and two drift spaces, rotates with the gantry, but remains on axis. It matches the round beam at the end of the transfer line to the gantry cells at any rotation angle. The beam emittances $\varepsilon_{x}=\varepsilon_{y}$ are assumed to be the same for both planes. Constraints for matching are equal $\beta$-functions, $\alpha_{x}=\alpha_{y}=0$, and $D_{x}=D_{x}^{\prime}=0$ at the entrance of the matching cell, and $\beta$-functions as in the gantry cell, $\alpha_{x}=$ $\alpha_{y}=0$, and $D_{x}=D_{x}^{\prime}=0$ at the exit of the matching cell.

\section{E. Ray tracking in the gantry}

The NS-FFAG accelerator rings, previously reported [5], have momentum ranges of $\delta p / p= \pm 50 \%$. The additional constraints at the central momentum for zero dispersion $D_{x}=0$ and $D_{x}^{\prime}=0$ would make the available energy range for the ring smaller, thus a larger value of the negative bend angle is needed for the gantry with respect to the NS-FFAG accelerator rings. The "swing" after roughly a quarter of the ring to the opposite bending direction makes shifts in the orbits at the end of the beam

TABLE I. Gantry optimization with respect to the bending angles.

\begin{tabular}{lcccccccc}
\hline \hline$\theta_{2}$ & $\theta_{1}$ & $\left|\theta_{2}\right| / \theta_{1}$ & $D_{\min }(m)$ & $x_{\min }(\mathrm{mm})$ & $x_{\max }(\mathrm{mm})$ & $\Delta x(\mathrm{~mm})$ & $x_{\min }^{\prime}(\mathrm{rad})$ & $x_{\max }^{\prime}(\mathrm{rad})$ \\
\hline 0.3332 & -0.08807 & 0.2643 & -0.00294 & -5.217 & 5.141 & 10.36 & -0.0081 & 0.02653 \\
0.3250 & -0.08397 & 0.2584 & -0.00138 & -4.942 & 4.445 & 9.387 & -0.0098 & 0.02353 \\
0.3193 & -0.08111 & 0.2540 & -0.0003 & -4.734 & 3.987 & 8.721 & -0.0111 & 0.02137 \\
0.3086 & -0.07578 & 0.2454 & 0.00175 & -4.303 & 3.449 & 7.752 & -0.0138 & 0.01733 \\
0.3046 & -0.07373 & 0.2421 & 0.00253 & -4.120 & 3.597 & 7.717 & -0.0149 & 0.01280 \\
0.3005 & -0.07168 & 0.2386 & 0.00332 & -3.927 & 3.733 & 7.660 & -0.0160 & 0.01427 \\
0.2964 & -0.06964 & 0.2350 & 0.00411 & -3.857 & 3.722 & 7.579 & -0.0172 & 0.01278 \\
0.2923 & -0.06759 & 0.2313 & 0.00490 & -3.507 & 3.970 & 7.477 & -0.0184 & 0.01162 \\
$\mathbf{0 . 2 8 4}$ & $\mathbf{- 0 . 0 6 3 5}$ & $\mathbf{0 . 2 2 3 5}$ & $\mathbf{0 . 0 0 6 4 9}$ & $\mathbf{- 3 . 0 4 2}$ & $\mathbf{4 . 2 2 7}$ & $\mathbf{7 . 2 6 9}$ & $\mathbf{- 0 . 0 2 0 9}$ & $\mathbf{0 . 0 1 2 9 8}$ \\
0.2800 & -0.06145 & 0.2195 & 0.00729 & -2.794 & 4.513 & 7.307 & -0.0222 & 0.13650 \\
0.2759 & -0.05940 & 0.2153 & 0.00808 & -2.535 & 4.800 & 7.335 & -0.0234 & 0.01432 \\
0.2677 & -0.05530 & 0.2066 & 0.00968 & -1.989 & 5.374 & 7.363 & -0.0261 & 0.01563 \\
\hline \hline
\end{tabular}




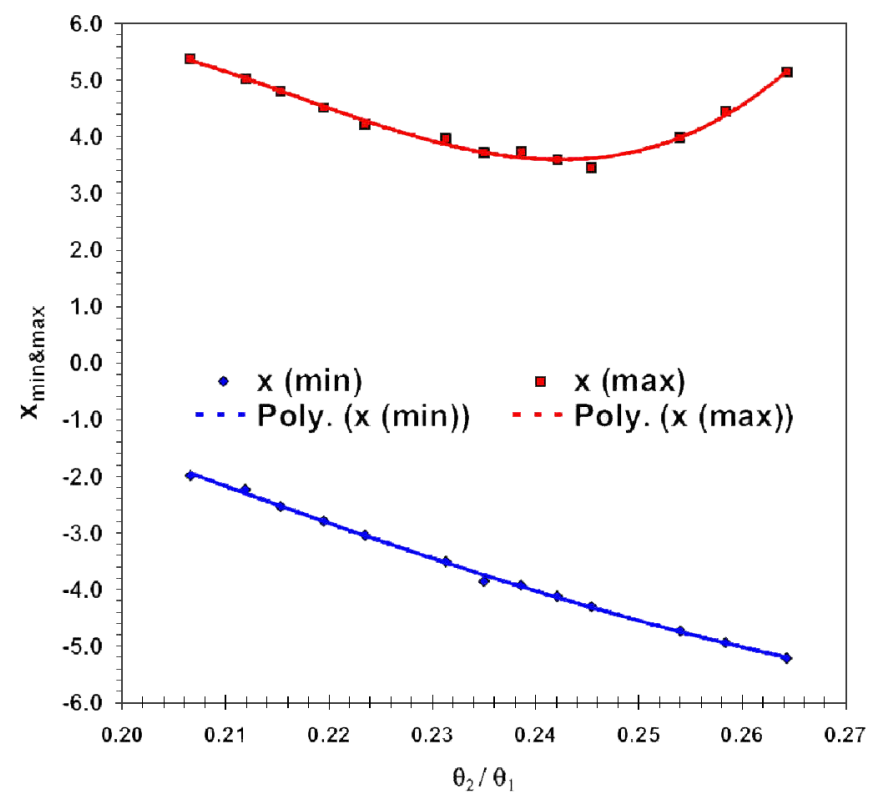

FIG. 6. (Color) Maximum orbit offsets dependence on $\theta_{B F} / \theta_{B D}$ at the end of the gantry.

line. The betatron functions are matched between the accelerator and the gantry only at the central momentum. Tracking is performed with initial conditions in both planes corresponding to a realistic beam size with maximum offsets of $3 \mathrm{~mm}$. Initial values of $x, x^{\prime}$ and $y, y^{\prime}$ at the middle of the central bend, where $\beta_{x}=0.3 \mathrm{~m}$ and $\beta_{y}=1.9 \mathrm{~m}$, are shown in Fig. 7.

Tracking results in the $\left(x, x^{\prime}\right)$ plane, at the end of the NSFFAG structure, are presented in Fig. 8 , in the $(x, y)$ plane in Fig. 9, and for the $\left(y, y^{\prime}\right)$ plane in Fig. 10. The orbit offsets at the end of the line vary between $\pm 6 \mathrm{~mm}$. There are 11 ellipses obtained from tracked particles (each labeled by corresponding marker) corresponding to the momentum range of $-25 \% \leq \delta p / p \leq 30 \%$ with steps

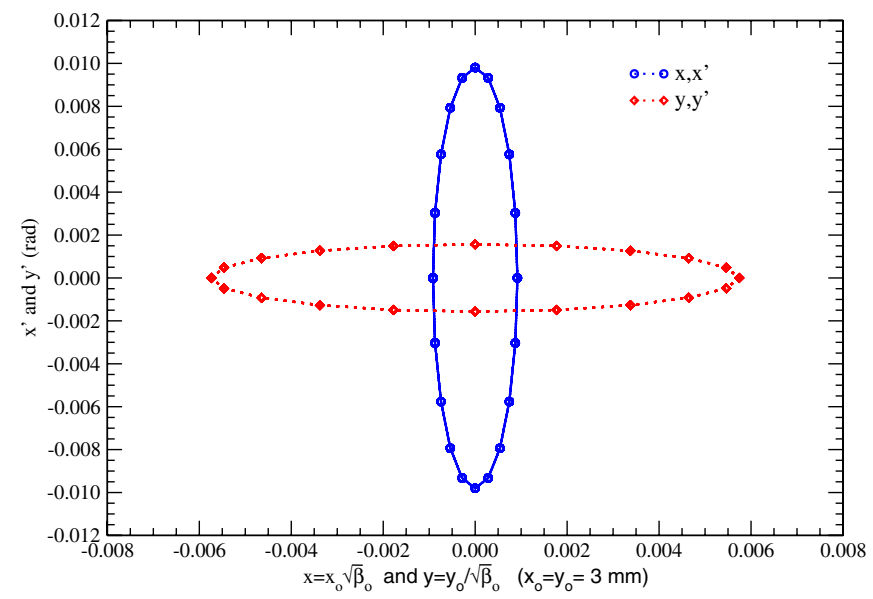

FIG. 7. (Color) Initial conditions for particles in the $\left(x, x^{\prime}\right)$ and $\left(y, y^{\prime}\right)$ phase space.

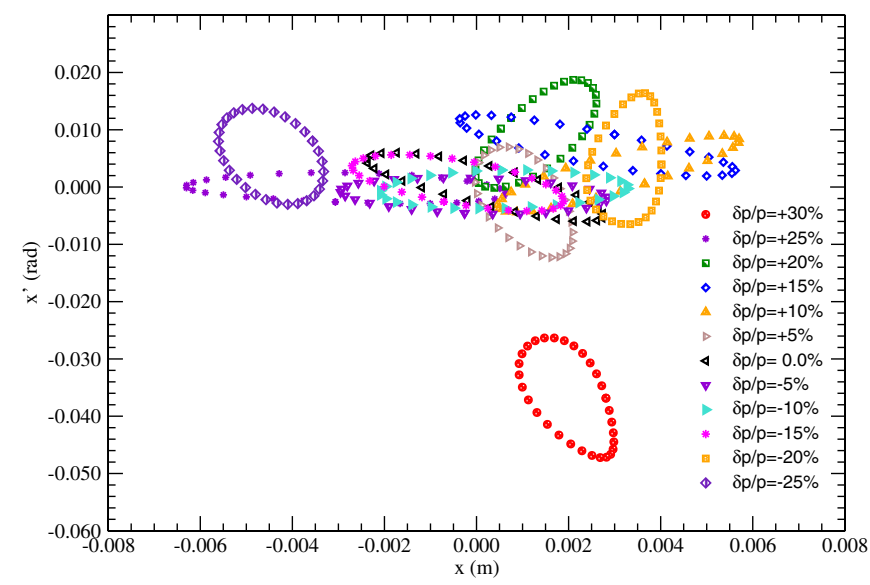

FIG. 8. (Color) Tracking results in $\left(x, x^{\prime}\right)$ phase space at the end of the nonscaling FFAG gantry.

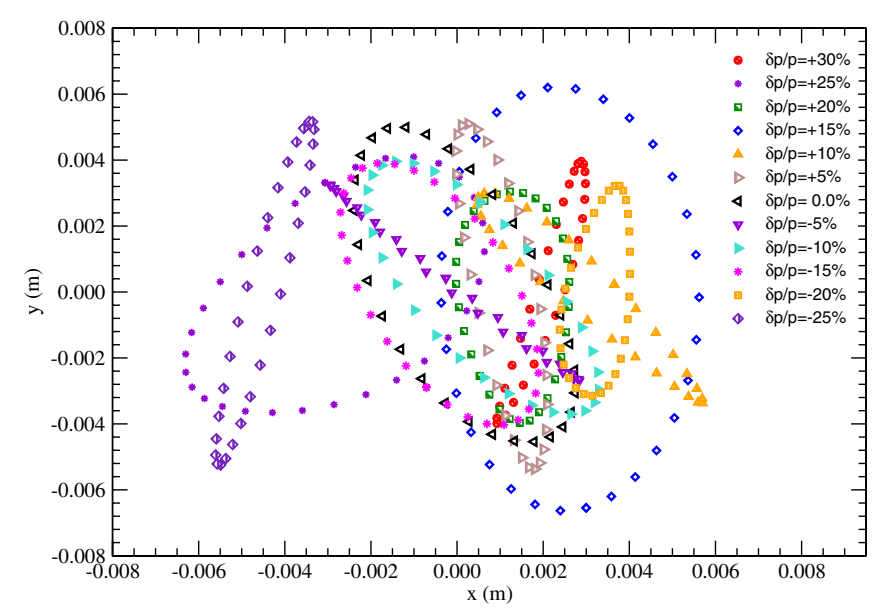

FIG. 9. (Color) Tracking results in $(x, y)$ phase space at the end of the nonscaling FFAG gantry.

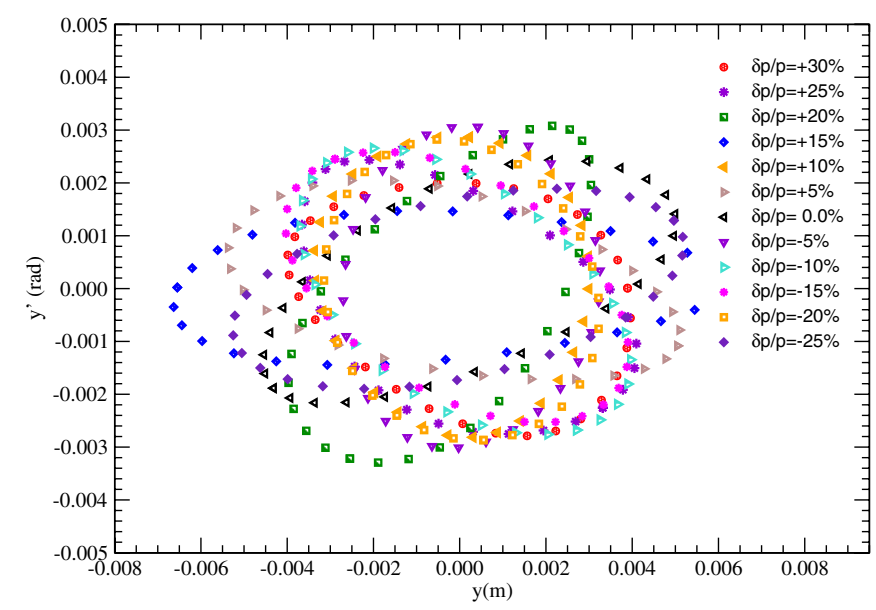

FIG. 10. (Color) Tracking results in $\left(y, y^{\prime}\right)$ phase space at the end of the nonscaling FFAG gantry. 
TABLE II. Magnet properties.

\begin{tabular}{lccccc}
\hline \hline Magnet & $L(\mathrm{~m})$ & $B(\mathrm{~T})$ & $G(\mathrm{~T} / \mathrm{m})$ & $A_{p}(\mathrm{~mm})$ & $B_{\max }(\mathrm{T})$ \\
\hline$B D$ & 0.40 & $3.4-3.9$ & 52.6 & $-1.5 \leq x \leq 6.9$ & 3.9 \\
$B F$ & 0.40 & $1.4-2.8$ & 54 & $-8.2 \leq x \leq 19$ & 2.8 \\
\hline \hline
\end{tabular}

in momentum of $\delta p / p=5 \%$. The quadrupole gradients in the following final focus triplet structure need to be adjusted for other than "central" energy with $\delta p=0$. Acceptance of the triplet quadrupoles at the $\delta p=0$ is within $\delta p / p \pm 1 \%$.

The magnetic field of the gantry magnets is fixed for the whole required energy range necessary for the treatment of any particular patient. The energy range of the tracking through the gantry is $149.7 \mathrm{MeV} / u \leq E_{k} \leq 400 \mathrm{MeV} / u$ or in momentum $-25 \% \leq \delta p / p \leq 30 \%$.

\section{F. Focusing and scanning magnets}

Ion beams reach the patient going through the focusing and scanning magnets at the end arc. The size of the beam spot at the patient at each energy is adjusted with the triplet quadrupole magnets, which have a separate control. The amplitude of the betatron functions could be set to the value of $\beta_{x}, \beta_{y}=0.35 \mathrm{~m}$. For standard operation we have chosen the value of $\beta_{x}, \beta_{y}=0.5 \mathrm{~m}$. The normalized emittance of the beam is assumed $\varepsilon_{n}=0.5 \times 10^{-6} \pi \mathrm{m}$. This will make the spot size at the smallest carbon energy of $E_{k}=149.7 \mathrm{MeV} / u$ or $\delta p / p=-25 \%$ equal to $0.65 \mathrm{~mm}$. Scanning in the plane of the patient bed is provided by two dipoles before the triplet quadrupoles. Different beam positions and beam entry angle are thus provided at each energy.

\section{G. Gantry magnets}

There are 28 NS-FFAG cells (about $0.92 \mathrm{~m}$ long) in the gantry. The same number of major bends-combinedfunction magnets is required. Magnet dimensions, magnetic field $B$ for the carbon energy ranging from 149 to $400 \mathrm{MeV} / u$ (see other details in Fig. 1) gradients $G$, and maximum aperture $A_{p}$, are summarized in Table II.

The required fields do not seem excessive for superconducting magnets. In order to save weight, we have investigated building them in a configuration with a simple inner quadrupole surrounded by a thicker outer dipole coil and a very thin dipole coil (active shield) at much larger radius. The cryostat has an $O D=170 \mathrm{~mm}$, and around the whole magnet is a thin warm iron shell to take care of the field not caught by the shield coil. The estimated weight of the magnets is in the range $45-75 \mathrm{~kg} / \mathrm{m}$ or about $50 \mathrm{~kg} / \mathrm{m}$ on the average. The gantry beam line is about $30 \mathrm{~m}$ long. It weighs about $1500 \mathrm{~kg}$.

\section{CONCLUSIONS}

An isocentric NS-FFAG gantry for the carbon/proton therapy, with fixed magnetic field for the whole energy range required for the patient treatment, is presented. The design has small magnet sizes and could reduce the gantry transfer elements weight, ease the gantry operation, and reduce the gantry cost. The transverse and final focus scanning system is assumed to be at end of the gantry above the patient. The kinetic energy range for the carbon cancer treatment of $150-400 \mathrm{MeV} / u$ requires settings of the fixed magnetic fields at the momentum rigidity of $B \rho=4.882 \mathrm{Tm}$, while for the proton treatment, with kinetic energy range of 79-250 MeV, the gantry magnets need to be set to the fixed magnetic fields at the momentum rigidity of $B \rho=1.87087 \mathrm{Tm}$. This work has been submitted for patent approval.

\section{ACKNOWLEDGMENTS}

We give special thanks to Etienne Forest for the Polymorphic Tracking Code special developments. The work of D. Trbojevic and B. Parker is supported by the U.S. Department of Energy under Contract No. DE-AC0298CH10886. The work of A. M. Sessler was supported by the U.S. Department of Energy under Contract No. DEAC02-05CH11231.

[1] U. Amaldi, Physica Medica XVII 1, 33 (2001), http:// www.physicamedica.com/VOLXVII_S1/09-AMALDI. pdf.

[2] J. Lindkvist, M. Ekman, S. R. Ericsson, B. Jonsson, and B. Glimelius, Acta Oncologica 44, 850 (2005).

[3] U. Weinrich, EPAC'06, Edinburgh, UK, 2006, p. 964.

[4] D. Trbojevic, E. D. Courant, and M. Blaskiewicz, Phys. Rev. ST Accel. Beams 8, 050101 (2005).

[5] E. Keil, A. Sessler, and D. Trbojevic, EPAC'06, Edinburgh, UK, 2006, p. 1681.

[6] T. Ohkawa (private communication).

[7] A. Kolomensky, Zh. Eksp. Teor. Fiz. 33, 298 (1957).

[8] K. R. Symon, D. W. Kerst, L. W. Jones, L. J. Laslett, and K. M. Terwilliger, Phys. Rev. 103, 1837 (1956).

[9] Y. Mori, EPAC'06, Edinburgh, UK, 2006, p. 950.

[10] E. Forest, E. Mcintosh, and F. Schmidt, KEK Report No. 2002-3, CERN-SL-2002-044 (AP), 2002), Vol. 44, p. 3. 